\title{
Téoros
}

Revue de recherche en tourisme

\section{Désir de nature et invention de la forêt en Occident}

\section{Gérard Beaudet}

Volume 25, numéro 3, automne 2006

Tourisme et forêt, visions d'une gestion intégrée

URI : https://id.erudit.org/iderudit/1071013ar

DOI : https://doi.org/10.7202/1071013ar

Aller au sommaire du numéro

Éditeur(s)

Université du Québec à Montréal

ISSN

0712-8657 (imprimé)

1923-2705 (numérique)

Découvrir la revue

Citer cet article

Beaudet, G. (2006). Désir de nature et invention de la forêt en Occident. Téoros, 25(3), 6-13. https://doi.org/10.7202/1071013ar d'utilisation que vous pouvez consulter en ligne.

https://apropos.erudit.org/fr/usagers/politique-dutilisation/ 


\section{Désir de nature et invention de la forêt en Occident}

\section{Gérard Beaudet}

" Je rêve d'un peuple qui commencerait par brûler les clôtures et laisser croître les forêts. "

Henry David Thoreau, De la marche.

Poumon de la planète, habitat, bassin de ressources ligneuses renouvelables, source d'énergie et de nourriture, complément de la culture et des élevages, la forêt est aussi, souvent et de plus en plus, territoire refuge, lieu de détente et espace de loisir.

Cette vision utilitaire de la forêt n'épuise cependant pas le rapport que l'Occident entretient avec ce milieu. La forêt est en effet le terreau d'un imaginaire et le refuge d'une mémoire collective qui contribuent encore et toujours à définir notre rapport à la nature (Harrison, 1992 ; Larrère et Nougarède, 1993 ; Schama, 1999).

Cette multiplicité de statuts, rarement exclusifs mais souvent difficilement conciliables, n'est évidemment pas donnée une fois pour toutes. La forêt, comme l'ensemble des autres milieux constitutifs de l'habitat humain, est un construit dont on peut retracer les origines et l'évolution. C'est ce à quoi s'attarde le présent texte. Il s'agit en l'occurrence de montrer comment notre rapport à la forêt a bifurqué quelque part au tournant du XVIIIe au $X I X{ }^{e}$ siècle, faisant de celle-ci un lieu d'expression d'une quête touristique qui a aussi, et à la même époque, transformé notre relation à la haute montagne et au littoral.

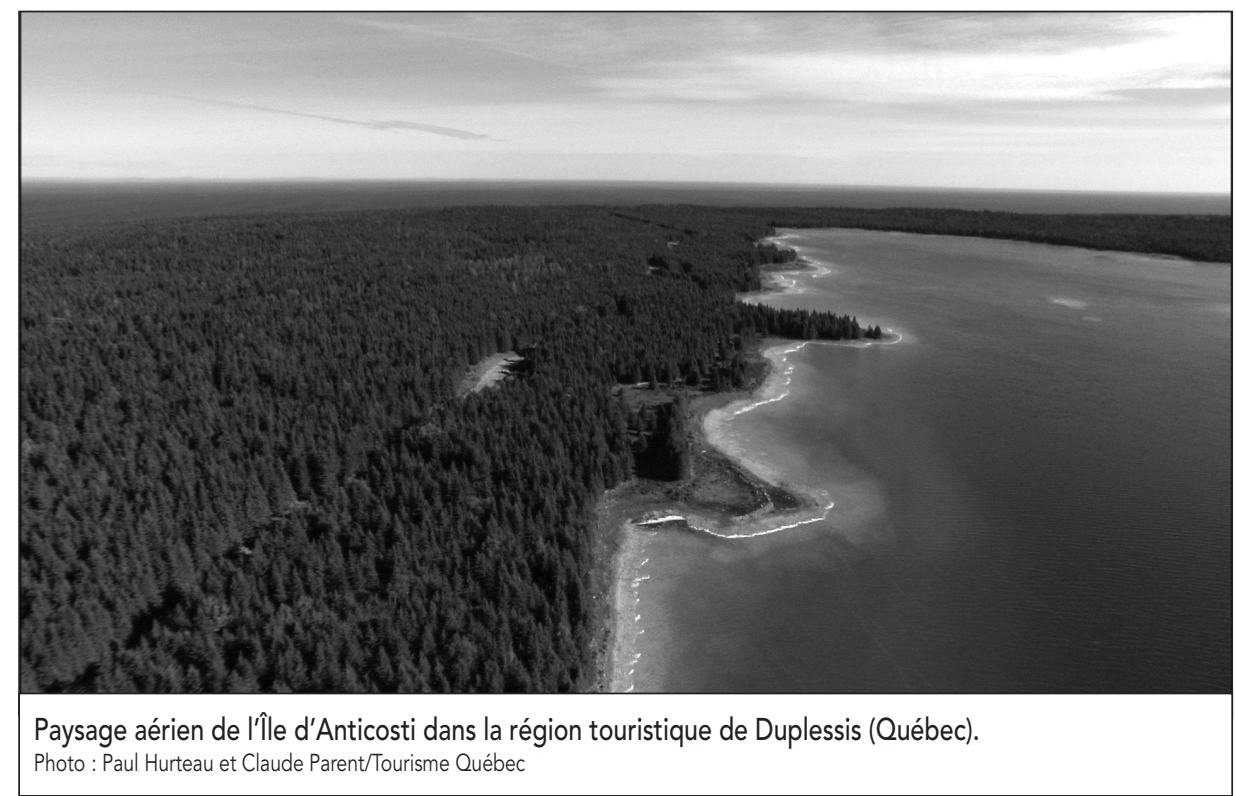

\section{La clairière, creuset d'un établissement humain précaire}

En Europe, le dégagement des premières clairières destinées à l'élevage et à la culture remonte à au moins 2000 ans av. J.C. Malgré l'amélioration de l'outillage et les accroissements démographiques, les massifs forestiers définiront toutefois encore longtemps I'horizon des habitats humains. Mais l'expansionnisme grec puis romain s'accompagnera d'un recul sans précédent de la sylve, particulièrement au pourtour de la Méditerranée.

La forêt, parfois ravagée jusqu'à engendrer des désordres environnementaux permanents ${ }^{1}$, n'est toutefois jamais totalement soumise. Le spectre de la reconquête sylvestre en effet hante longtemps les communautés confrontées à l'effondrement de leur économie, à l'érosion de leurs institutions ou au déclin de leur démographie.
Même si une telle reconquête est surtout le lot des franges de l'établissement, l'image de la ville en ruine littéralement absorbée par une forêt luxuriante ne nourrit pas notre imaginaire collectif à la seule faveur des grandes découvertes archéologiques du XIXe siècle, notamment en Amérique centrale, en Amérique du Sud et en Asie du Sud-Est. Capitale d'un vaste empire déchu, même l'orgueilleuse Rome impériale aura été victime d'une telle reconquête:

Tandis que la cité se désintègre de l'intérieur, la forêt l'envahit de l'extérieur. L'antique cité de Rome [...] fut finalement reconquise par les forêts, d'abord par analogie, puis par des populations d'hommes des bois venus du Nord, et enfin par la ceinture de végétation elle-même (Harrison, 1992 : 33).

Mais, la forêt n'est pas menaçante que par ses seules avancées potentielles. Aux marges de l'Empire romain, les sombres massifs 
forestiers d'Europe du Nord marquent les limites de la civilisation et contiennent en germe le déferlement de la barbarie qu'ils abritent. Dans le haut Moyen Âge, "c'est là que vivaient les proscrits, les fous, les amants, les brigands, les ermites, les saints, les lépreux, les maquisards, les fugitifs, les inadaptés, les persécutés, les hommes sauvages " (Harrison, 1992 : 99). Dans les siècles suivants, les légendes, les contes et les récits mettant en scène sorciers, fées, ogres et animaux sauvages fondent la curiosité, la crainte, l'enchantement et la terreur indistinctement associés à une forêt où on s'aventure toujours à ses risques et périls.

De nos jours, notre désir de nature semble être l'aboutissement d'un parcours qui aurait conduit, à la veille de l'époque romantique, à une réconciliation avec la forêt, ou plutôt à un « réenchantement ». Mais pour que cette réconciliation soit possible, encore fallait-il que la forêt soit préalablement apprivoisée, c'està-dire, en l'occurrence, aménagée. Le « réenchantement » ne fut toutefois pas le résultat de cet aménagement ; il aura plutôt été engendré par ses excès puis en aura fondé et accompagné la contestation.

\section{La forêt interdite}

En Occident, l'établissement humain s'est construit au détriment de la forêt. Le dégagement d'une clairière est en effet, plus souvent qu'autrement, la condition de possibilité de l'installation de populations sédentaires.

Territoire du sacré, lieu d'une terreur jamais complètement apaisée, refuge du paganisme, espace réservé et par conséquent interdit, la forêt agit longtemps et diversement comme un lieu dont l'attractivité est contenue par des restrictions d'occupation permanente imposées ou consenties, qui repoussent à ses marges l'établissement. Ce faisant, la forêt participe d'une catégorisation de l'espace géographique en domaines qualitativement différenciés. Elle agit en d'autres termes comme un vacuum, soit

un domaine vide qui discontinue l'écoumène en devenir, parce que frappé d'un interdit d'établissement permanent. Ce vacuum primitif doit être vaste, d'une superficie de quelques centaines de kilomètres carrés au minimum. II répartit sur son pourtour la population concernée en diverses communautés de telle ma- nière que leurs rivalités puissent être dispersées au loin. La distance produite par l'extension du grand vacuum introduit ainsi un écart différentiel constitutif à la fois d'identité et d'altérité (Desmarais, $2006: 51)$.

Consubstantielle de la diversité culturelle de l'Europe, la forêt se dresse en quelque sorte face à la conquête romaine, dont les desseins et le dessin s'articulent à un espace indifférencié et homogénéisé par les découpages institutionnels (provinces, centuries) et les occupations de surface (routes, aqueducs, villes, villas, etc.).

En se retournant contre sa matrice et en partant à la conquête du monde, l'administration civile romaine partit aussi en guerre contre les grandes forêts du monde antique. De l'Angleterre à l'Italie en passant par la Gaule et l'Espagne, et dans tout le bassin méditerranéen, les forêts étaient partout. Leur densité hostile avait jadis garanti une relative autonomie aux familles et aux villes de l'Antiquité, en leur offrant comme un havre d'intimité culturelle. On peut dire qu'elles ont alimenté les diversités culturelles en procurant l'isolement nécessaire au surgissement d'une identité de langage, de coutumes, de divinités, de traditions, de styles, etc. Les forêts étaient des obstacles à la conquête, à l'hégémonie, à l'homogénéité, à l'homogénéisation. Elles étaient, en un mot, des refuges d'indépendance culturelle. (Harrison, 1992 : 86-87)

Si la déforestation s'est imposée, c'est au moins autant pour briser une des conditions de possibilité de la résistance des peuplades conquises que pour livrer, sous différentes formes, la ressource ligneuse et préparer le terrain pour l'élevage et la mise en culture. L'indifférenciation de surface de l'aire romanisée ne résiste cependant pas au recul de la civilisation romaine et on assiste, partout où l'érosion n'avait pas causé des dommages irréversibles, au retour de la forêt.

\section{La forêt et l'affirmation de la royauté médiévale}

La forêt acquiert, dès le haut Moyen Âge, un nouveau statut en lien avec l'affirmation progressive de la royauté. La mise en réserve de vastes étendues boisées, transformées en sanctuaires de la vie sauvage, permet en effet le renforcement de ce rituel fondateur que constitue la chasse (Harrison, 1992). Créée dans le Hampshire à la fin du Xle siècle par Guillaume le Conquérant, à la faveur de la confiscation de nombreuses terres, de la destruction de villages et du déplacement de populations, la New Forest reste une des plus célèbres de ces initiatives lancées par les souverains ${ }^{2}$. D'une superficie de plus de 400 kilomètres carrés, elle fondera, comme plusieurs autres forêts royales en GrandeBretagne et en Europe continentale, une catégorisation qualitativement différenciée de l'espace féodal qui s'est avérée passablement durable ${ }^{3}$. En effet,

on peut considérer ces rois-chasseurs comme les premiers conservateurs publics ou institutionnels de l'histoire. Si les forêts au sens juridique n'avaient pas été inventées au Moyen Âge, les forêts au sens naturel auraient sans doute depuis longtemps entamé leur disparition de l'Europe civilisée. (Harrrison, 1992 : 112)

Si certaines de ces réserves, à l'instar de la forêt de Windsor ou de Richmond Park - le plus grand parc urbain d'Europe - en Angleterre, sont toujours des propriétés royales, la plupart sont devenues, particulièrement au XIXe siècle, des propriétés publiques. Dorénavant accessibles à la population, plusieurs de ces anciennes forêts royales sont maintenant de grands parcs métropolitains - par exemple la forêt de Vincennes et les parcs du Bois-de-Boulogne et de Versailles à Paris -, dont profitent résidants et touristes. D'autres, comme la forêt de Biatowieza, qui s'étend sur plus de 150000 hectares à la frontière de la Pologne, du Bélarus et de la Lituanie et est devenue réserve mondiale de la biosphère, comptent parmi les rares habitats sauvages qui aient survécu en Europe.

Si la création de ces réserves s'accompagne de préoccupations pour la conservation et le renouvellement de la ressource ligneuse, il faudra attendre la Renaissance pour que s'instaure un nouveau rapport à la nature susceptible de fonder, à terme, une véritable science de la forêt.

\section{La forêt tirée au cordeau}

Amorcée à la Renaissance, la transformation du rapport Homme-Nature s'accélère avec les Lumières. En logeant le paradis de la Divine Comédie (v. 1306-1321) dans une forêt antique débarrassée des dangers et des 
pièges de l'obscure forêt dans laquelle il s'était initialement égaré, Dante préfigure en quelque sorte Descartes, qui réclame la coIonisation systématique d'un monde ensauvagé et superstitieux. La nature devrait désormais se plier aux impératifs de la raison.

Cette révolution prend appui sur le développement de connaissances sur l'environnement physique, le recours à de nouveaux instruments facilitant les relevés, la diffusion de traités - notamment sur les jardins et les fortifications - et la réalisation de parcs, qui alimentent au XVII ${ }^{\text {e }}$ siècle un désir d'ordonnancement de la ville et de la campagne. L'aménagement du territoire est né (Mariage, 2003). La forêt n'y échappe pas.

Malgré la lourdeur des peines encourues par quiconque défie les nombreux interdits, l'ensemble des massifs forestiers d'Europe subit des dégradations de toutes sortes, en particulier aux XIVe, XVe et XVI ${ }^{e}$ siècles. Construction navale ou résidentielle, chauffage, fabrication du verre et de briques, de même que métallurgie, requièrent en effet des prélèvements d'une ampleur telle que plusieurs pays s'inquiéteront, au XVIIe siècle, pour l'avenir de leurs flottes navales et de leur économie en général. La situation est tellement préoccupante qu'il ne suffit plus de restreindre par réglementation ces prélèvements. II faut impérativement augmenter la productivité des forêts ${ }^{4}$. Or, pour ce faire, cette dernière doit être pensée autrement, c'est-à-dire comme ressource plutôt que milieu.

L'adoption par Colbert, en 1669, d'une ordonnance qui constitue un véritable code forestier et le développement d'une sylviculture scientifique, dans l'Allemagne du $\mathrm{XVIII}$ e siècle, confirment, chacun à leur façon, cette réduction de la forêt à des volumes de bois exploitables. Les forestiers n'ont dès lors de cesse de perfectionner savoirs et méthodes. La forêt, littéralement mise en ordre, est désormais soumise à une exploitation rationnelle destinée à garantir la qualité et la pérennité de l'approvisionnement. La mutation est telle qu'au XIX ${ }^{e}$ siècle, «[la] forêt germanique devi[e]nt l'archétype de la soumission d'une nature désordonnée aux constructions rigoureuses de la science... » (Harrison, 1992: 187). Tirées au cordeau, débarrassées de leurs espèces moins nobles ou moins productives, soumises à des pratiques sylvicoles diverses et exploitées en fonction de savants calculs, les forêts deviennent le domaine exclusif des ingénieurs forestiers. Économie rurale et économie forestière sont résolument dissociées. Cette volonté d'administrer scientifiquement la production sylvicole a un prix ; la forêt est dorénavant " désenchantée » (Larrère et Nougarède, 1993).

À la même époque au Québec, comme dans l'ensemble du Nord-Est américain, la forêt, longtemps restée le territoire incontesté des Amérindiens, recule devant les défricheurs qui abandonnent les rivages des cours et des plans d'eau pour repousser vers l'intérieur des terres les limites de l'écoumène ${ }^{5}$. Mais l'exploitation forestière ne se justifie pas par le seul désir de faire de la terre. Confrontée au blocus napoléonien qui lui interdit, à compter de 1801, l'accès au bois de la Baltique, l'Angleterre se tourne vers sa colonie outre-Atlantique pour approvisionner ses chantiers navals. Les bûcherons se lancent dès lors à l'assaut des massifs de grands pins, notamment ceux de l'Outaouais et de la Mauricie, qu'ils décimeront presque complètement. Ici, la sylviculture sera de peu d'intérêt face à l'immensité en apparence inépuisable des forêts, qu'au demeurant on doit abattre pour faire sa place. Nostalgie et rêverie ne sont pas (encore) de mise.

\section{La représentation urbaine de la nature}

À la Renaissance, l'invention de la peinture de paysage, la Réforme et la découverte du Nouveau Monde marquent durablement l'imaginaire européen du rapport au monde et seront ultimement à l'origine de «l'idée de créer des espaces dédiés à la nature, sortes de jardins d'Éden sauvés de l'homme, figures, pour l'au-delà des temps, de l'avant " (Viard, 1990 : 36). La création de ces sanctuaires aujourd'hui vénérés par les touristes est toutefois le résultat d'un long cheminement marqué du sceau du romantisme.

À l'instar de la rupture de la transcendance qui révèle, à la Renaissance, une contestation des formes traditionnelles de l'autorité de la chrétienté médiévale, l'insurrection romantique signale une remise en cause du culte de la Raison (Beaudet, 1999). La montagne, le littoral et la forêt deviennent en quelque sorte les territoires de prédilection de l'expression de la contestation romantique. Mais cette quête de nature transposée au loin est précédée par une représentation en rapproché de celle-ci. Le phénomène n'est pas inédit puisque, de tout temps, la nature a été " 'représentée', convertie en espace vert par exemple, là où elle manque, là où elle est communiquée par le langage, c'est-à-dire en pleine position urbaine » (Ritchot, 1998 : 73). En effet,

[la] nature est depuis toujours un matériau urbain. Déjà l'antique Babylone cultivait l'art du jardin, tout comme New York qui, au siècle dernier, souda son destin à celui de Central Park. Encore aujourd'hui, il paraît impossible, en Occident du moins, de concevoir l'urbanisme et l'urbanisation sans la construction de parcs, de squares et de jardins. Autant d'espaces où de précises mises en scène donnent à voir l'un ou l'autre des éléments naturels: I'arbre, l'herbe et la fleur, la montagne et la plaine, l'eau et le ciel. (Mercier, 1998 : 239)

Paradoxalement, cette quête de nature est d'emblée nourrie par un héritage des Lumières. C'est en effet à la faveur de la montée des préoccupations hygiénistes que l'espace vert s'impose comme solution de rechange à la tenue en échec de la médecine par la maladie. En France, l'aménagement de cours et de promenades dans les emprises dégagées des anciennes fortifications inaugure ce mouvement dès la fin du XVIII siècle. À Londres, l'ouverture au public de St. James's Park sous Charles II anticipe ce fleuron de l'urbanisme géorgien que constituera le square.

Mais, si cette représentation de la nature permet la régénération du corps et de l'âme des élites urbaines, elle ne parvient pas à apaiser les tourments de ceux qui remettent en cause la conquête de la nature par les Lumières (Viard, 1990). Pour ces derniers, la forêt constitue un havre de solitude propice à l'introspection. Déjà, au XIVe siècle, l'écrivain italien Pétrarque trouve refuge dans la forêt de Laure pour s'y adonner à la rêverie. Mais c'est sans conteste Jean-Jacques Rousseau qui donne à cette évasion ses lettres de noblesse.

\section{Les romantiques et le "réenchantement " de la forêt}

En se promenant seul au bois de Boulogne, Rousseau inscrit ses réflexions en marge de la société et de la cité, dont il conteste les prétendus bienfaits. Certes, la forêt de Saint-Germain n'est plus la forêt primitive 
des temps anciens. Pourtant, l'affinité naturelle entre cette forêt apprêtée et son lointain modèle permet à Rousseau d'entrevoir l'état de nature. Là, sous les grands arbres, à l'ombre de la lumière de la raison, l'intuition s'allie à l'environnement suggestif de la forêt pour atteindre, au-delà du temps, au-delà de l'histoire et de ses institutions, la vérité de "l'homme naturel " dans son rapport intime à ses origines naturelles. (Harrison, 1992: 196)

Nourri de nostalgie, le pré-romantisme contribue à un "réenchantement » de la nature. Mais, celle-ci n'est pas appréhendée en mode naturaliste. Elle est plutôt l'objet d'une double artialisation (Roger, 1997). Alors que peintres et écrivains réalisent d'emblée une artialisation in visu, les aménageurs élaborent une artialisation in situ.

La randonnée est indissociable de cette double artialisation. Bien que d'abord motivées par un intérêt scientifique, les expéditions du poète-géologue suisse Horace-Bénédict de Saussure (1740-1799) au mont Blanc et dans les massifs du Jura, des Vosges ou du Vivarais se doublent de préoccupations esthétiques. La diffusion de ses écrits contribue à la création de la montagne et à sa mise en tourisme ultérieure. En Angleterre, William Wordsworth (1770-1850) et ses compagnons transforment « l'idée même de la marche, et fond[ent] de ce fait la longue lignée de ceux qui la pratiquent pour le double plaisir d'avancer sur deux jambes et de s'imprégner du paysage à la source de leur inspiration » (Solnit, 2002 : 113). Ces romantiques de la première heure "ont réinventé la marche en tant qu'activité culturelle participant pleinement de l'expérience esthétique » (ibid.). D'autres œuvrent à populariser cette activité, dont l'Allemand Karl Baedeker (1801-1859) qui publie, sous forme de guides, le compte rendu de ses excursions pédestres.

\section{Fontainebleau et I'artialisation de la nature}

En France, la forêt de Fontainebleau est le terrain privilégié et inaugural d'une telle artialisation. Ce massif de quelque 25000 hectares, situé à une soixantaine de kilomètres de Paris, devient, en deuxième moitié du XIX ${ }^{e}$ siècle, un des lieux de fondation du tourisme en forêt (Boutefeu, 2005 ; Kalaora, 1998). Le lieu est d'abord fréquenté par des artistes, dont les peintres de l'École dite de Barbizon qui, en posant leur regard sur la nature, l'ont immortalisée. Par leur art, mais aussi par leurs pratiques, le paysage forestier est entré dans le cercle de la sensibilité collective : ils ont cristallisé des significations, des expériences, des sensations, des réminiscences, des impressions. [...] La forêt de Fontainebleau devint un nouveau salon, une "promenade parisienne » que fréquente un public fortuné soucieux de rehausser son prestige et son statut. (Kalaora, 1998 : 21-22)

Claude François Denecourt (1788-1875), militaire retraité et autodidacte, se fixe à Fontainebleau en 1832 et fait de la forêt son refuge sentimental. Mais, contrairement à Rousseau et à d'autres promeneurs solitaires, il souhaite faire partager sur le terrain une passion alimentée par des sensibilités artistiques :

le tableau d'une nature aussi grandiose doit être l'œuvre du peintre ou du poète, dont elle enflamme le génie, et [...] ma mission doit se borner à diriger le voyageur qui vient en admirer les beautés. Attiré moi-même par la douce paix et le bonheur qui résident au fond de ces bois et de ces déserts, je les ai longtemps parcourus, et, aidé par les observations des artistes qui les fréquentent journellement, j'ai acquis une connaissance de la localité qui m'a mis à même d'en signaler toutes les parties les plus pittoresques, et d'offrir au voyageur les moyens de les visiter avec autant de facilité que d'agrément. ([http://www.aaf.org])

Aussi Denecourt ne se contente-t-il pas de faire connaître le lieu ; il publie un guide en 1839 et trace un premier sentier de promenade en 1842. L'inauguration d'un chemin de fer en 1849 favorise la fréquentation de la forêt par les Parisiens qui consacrent l'avènement d'une «Forêt-Loisir » (Kalaora, 1998).

L'image populaire et littéraire de la forêt hostile, associée aux ténèbres, à l'obscurité, à l'état originel de nature, fait place à celle d'une paisible zone d'accueil pour l'élite sociale qui, durant la seconde moitié du XIXe siècle, s'emploiera à en établir les règles de consommation et d'usage qui serviront de modèle de référence pour son aménagement récréatif. (Kalaora, $1998: 21$ )
Cette transformation des sensibilités porte en germe la contestation d'un modèle productiviste et industriel de la foresterie. Dès 1830, des artistes qui fréquentent Fontainebleau s'opposent à la plantation de 6000 hectares de résineux. En 1861, l'abattage des arbres y est par ailleurs formellement interdit sur des séries artistiques. II s'agit de la première mesure de protection de la nature. Celle-ci s'articule d'emblée à une valorisation esthétique plutôt qu'à une caractérisation scientifique du milieu.

\section{L'invention de la forêt romantique}

À cette époque charnière, la création de la forêt se décline également sur d'autres modes. En Allemagne, la publication de recherches et les écrits d'inspiration folklorique des frères Grimm participent, dans le premier quart du XIXe siècle, d'une ressaisie de l'âme nationale dont les forêts ont été le terreau et le refuge.

Tout se passait comme si les vieilles forêts allemandes détenaient les vérités essentielles sur les coutumes, les lois et la culture - vérités susceptibles de mieux faire comprendre l'Allemagne [...] et d'unifier le peuple allemand à une époque où les principautés allemandes étaient divisées et occupées par les Français, au moment des guerres napoléoniennes (Zipes, cité dans Harrison, 1992 : 246).

Aux États-Unis, la publication en 1826 du roman Le dernier des Mohicans de James Fenimore Cooper (1992) conjugue la forêt en mode nostalgique. Situant l'intrigue au moment de la guerre d'Indépendance, l'auteur décrit en effet un environnement forestier qui a depuis été bouleversé par la colonisation, l'agriculture, l'urbanisation, l'industrialisation et l'exploitation forestière. Cela n'empêche cependant pas le poète et essayiste Henry David Thoreau (1817-1862) de trouver refuge dans les forêts du Maine quelques années plus tard pour s'y affranchir de la société et y mener une expérience spirituelle qu'il consigne dans son ouvrage Walden ou la vie dans les bois (1982). Précurseur de la pensée écologiste, Thoreau influencera le mouvement de communion avec la nature, qui est héritier en quelque sorte du transcendantalisme américain. La transformation du couvert forestier appalachien en paysages ne sera toutefois achevée qu'avec la construction d'un nouvel imaginaire forgé à la faveur de la découverte des forêts de la côte Ouest (Roger, 1997). 
C'est en effet le franchissement des Appalaches et la conquête de l'Ouest américain qui marquent aux États-Unis un véritable infléchissement du rapport à la nature et à la forêt. En 1852, alors que la Sierra Nevada est envahie par des hordes de journaliers qui travaillent aux mines de la région, un chasseur découvre les géants de la vallée de Yosemite. Trois ans plus tard, les premiers touristes arpentent les bois de Calaveras et y découvrent à leur tour cette forêt de séquoias. Photographies et gravures, dont celles de Carleton Watkins, exposées à New York en 1862, ont tôt fait de susciter un engouement qui pousse des habitants de la côte Est à entreprendre ce qui constitue un véritable pèlerinage. Mobilisé par ce que d'aucuns assimilent à un temple de la Nature, le mouvement en faveur de la protection de cette forêt s'intensifie, tant et si bien qu'Abraham Lincoln créé en 1864, en pleine guerre de Sécession, le premier parc naturel du monde (Schama, 1999: 312ss). L'initiative fera école.

Malgré des différences notables, attribuables entre autres à la nature des milieux forestiers et à leur histoire sociale, on assiste donc, en deuxième moitié du XIX ${ }^{e}$ siècle, au développement du loisir et du tourisme en forêt. Ce développement se heurte toutefois à une conception solidement ancrée qui fait de la forêt une source de matière ligneuse ou de bois d'œuvre et, spécialement en Europe, un moyen de protection contre l'érosion.

Une telle conception engendre « une vision hégémonique et normative de l'aménagement des forêts » (Boutefeu, 2005 : 8). C'est pourquoi les hauts lieux du tourisme et du loisir en forêt doivent habituellement être consacrés par l'attribution d'un statut qui révèle les valeurs culturelles, artistiques et esthétiques dont ils sont le gisement. En d'autres termes, ils doivent être soustraits, au moins en partie, à l'emprise de l'ingénierie sylvicole et des prélèvements à des fins économiques.

Si la plupart des pays occidentaux adoptent des lois en ce sens après 1880, les décalages dans le temps révèlent des écarts considérables dans la construction de la nouvelle sensibilité. Par ailleurs, malgré quelques avancées notables en matière de conservation, la forêt-refuge trouve difficilement son espace vital au sein du territoire de la forêt-ressource. Au Québec, la création des parcs de la Montagne-Tremblante (1894) et des Laurentides (1895) révèle cette subor- dination des objectifs qui sous-tendent la désignation de la première aux considérations économiques qui fondent la vocation de la seconde, puisque l'exploitation forestière y reste permise.

\section{L'usage social de la forêt}

La création de parcs de conservation et de réserves n'épuise évidemment pas les modalités de la satisfaction de la demande pour un usage social de la forêt à des fins de loisirs et de tourisme. Celle-ci se heurte toutefois, depuis ses premières manifestations, à un modèle productiviste de la gestion forestière. Mais cette demande n'est pas seule en cause.

En effet, en vertu du modèle productiviste qui s'est imposé au cours du XIX ${ }^{\circledR}$ siècle, la priorité donnée au rendement confère à l'espace forestier une vocation exclusive articulée à un compartimentage spatial qui pose cet espace en marge des établissements humains. En d'autres termes, la mise en ordre de la forêt déjà évoquée engendre progressivement une catégorisation spatiale ségrégative qui exclut la forêt de la géographie territoriale et sociale de la ruralité. La difficile conciliation entre les usages sociaux et économiques de la forêt se mue ainsi en incompatibilité quasi totale, particulièrement en Amérique du Nord, où les préoccupations environnementales tardent à infléchir les pratiques des forestiers.
Considéré comme un des fondateurs de la sociologie rurale, Frédéric Le Play (1806-1882) tente, par ses recherches et ses travaux sur le terrain, de contrer ce mouvement.

Le Play reli[e] [...] la question des forêts à la question sociale, et il trait[e] la sylviculture dans une perspective autant naturaliste que sociale. L'interaction naturesociété [est] au centre de ses travaux [...] en ce qui concerne plus particulièrement la question forestière, les liens entre société, sylviculture et nature. À ce titre, sa pensée, comparée aux conceptions ayant cours à son époque [apparaît] radicalement originale voire même annonciatrice, tant du point de vue de l'histoire économique et de l'histoire des sciences et des techniques (géographique, sylvicole, sociologique) que des théories écologiques modernes (Kalaora, 1998: 46).

Ayant fait école en France, Le Play aura également des disciples au Québec, dont Esdras Minville (1896-1975), qui s'inspire des travaux de celui-ci pour élaborer, à compter des années 1920, son projet d'économie sociale dans l'arrière-pays de Grande-Vallée en Gaspésie (Foisy-Geoffroy, 2004: 143ss). Mais, ici comme un peu partout en Occident, la demande sociale doit continuer à disputer son espace vital à celui revendiqué par les promoteurs de la forêt-ressource.

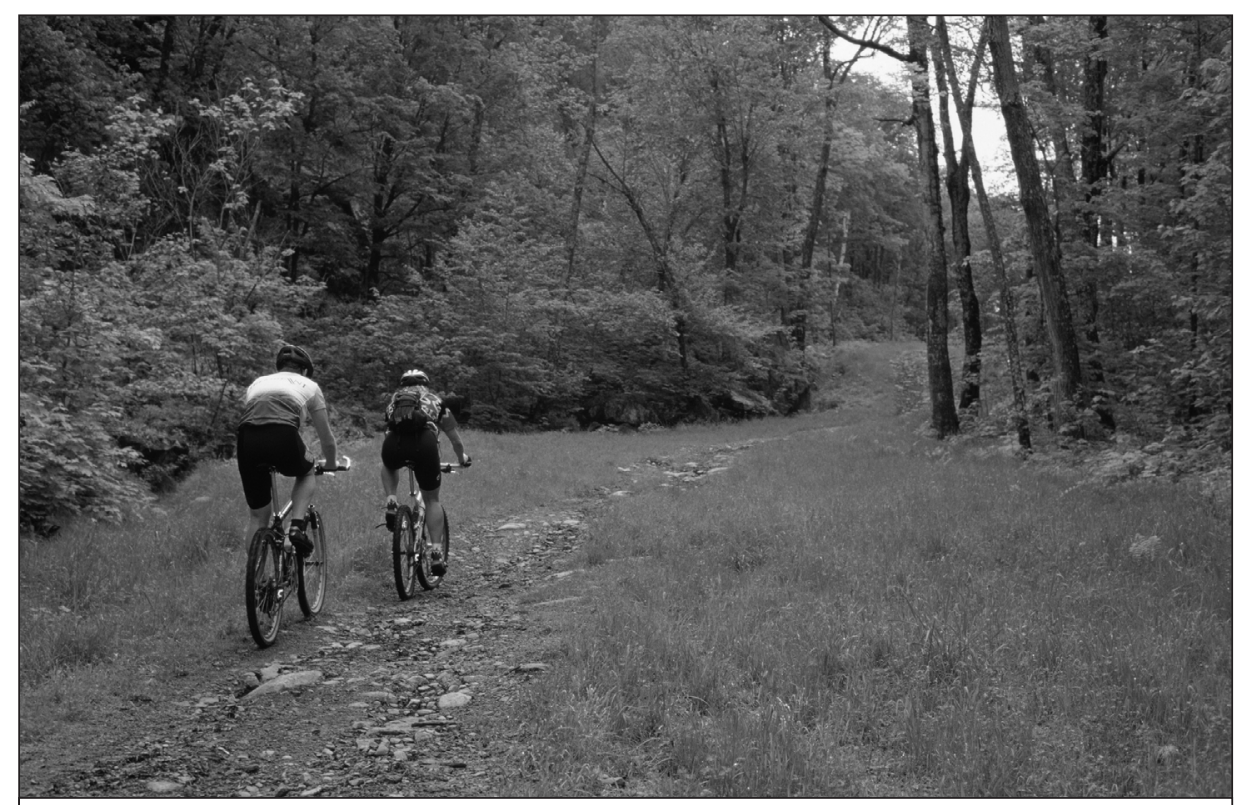

Cyclistes pratiquant le vélo de montagne dans la région touristique des Laurentides (Québec).

Photo: Michel Julien/Tourisme Québec 


\section{Du club de chasse au parc-nature : la démocratisation de la demande sociale au Québec}

Au Québec, la publication du poème Hymne au coureur des bois ${ }^{6}$ de l'abbé Henri-Raymond Casgrain (1831-1904) réhabilite ce personnage condamné par les autorités civiles de la Nouvelle-France et par l'Église, qui persiste en ce sens longtemps après la Conquête de 1760. L'euphorie de la liberté et du contact avec la nature qui fonde ce mythe (Germain, 2003) occulte désormais la licence des comportements. C'est qu'au Québec, comme du reste dans l'ensemble du Nord-Est américain, la nouvelle sensibilité à l'égard de la nature, née dans le sillage de l'expérience quasi religieuse de la découverte des géants de la côte du Pacifique, rejoint une certaine élite. Celle-ci développe cependant des usages qui diffèrent sensiblement de ceux qui avaient alors cours en Europe. Ici,

la mimésis à l'œuvre semble avoir posé le coureur des bois comme une figure emblématique du héros romantique. Contrairement au célèbre Montreal Hunt Club, qui proposait la chasse à courre dans la proche campagne du centre-nord de l'île, ou à la propriété Menier à Anticosti qui, pour sa part, évoque le modèle aristocratique du vaste domaine, les clubs de chasse et pêche qui sont apparus au début du XIXe siècle et se sont multipliés à compter de 1885 auraient davantage répondu de l'attrait pour le genre de vie caractéristique d'un nomadisme sélectif perdu, que pratiquaient jadis certains grand nomades des bois (Beaudet et Gagnon, 1999: 179).

Mais cette réhabilitation du coureur des bois ne signifie pas pour autant que ses descendants retrouvent le privilège de fréquenter la forêt autrement que pour y travailler dans les chantiers. En effet, l'accès à la forêt et aux meilleurs territoires de chasse est alors un privilège de classe. Seuls les nantis, au nombre desquels figurent surtout des Canadiens anglais et des Américains, peuvent se payer les droits exigés par les clubs auxquels le gouvernement accorde de vastes concessions. Inauguré en Mauricie en 1880 avec la fondation du club Winchester, ce système de chasses gardées perdurera jusqu'en 1977 (Martin, 1990 : 81ss). À cette époque, la multiplication du nombre de chasseurs et de pêcheurs rend politiquement difficile le maintien du privilège des clubs privés. Le « déclubage » contribue à la démocratisation de la chasse. Entre-temps, de nouvelles formes de loisirs et de tourisme en forêt se développent.
La création, en 1970, du parc national de la Mauricie sur un territoire de 536 kilomètres carrés témoigne de cette évolution. II ne s'agit plus seulement de favoriser la conservation de monuments de la nature ou de réserver un territoire à quelques privilégiés. II s'agit désormais de permettre l'accès à la nature au plus grand nombre, à des fins de loisirs variés, de la randonnée à l'observation de la faune, en passant par le camping familial et l'excursion en canot.

Le confinement inhérent au parc, aussi vaste soit-il, ne convient toutefois pas à tous. Par exemple, motoneigistes puis "VTTistes ${ }^{7}$ " souhaiteront avoir accès à de vastes réseaux de randonnée qui s'inscrivent, pour l'essentiel, en forêt. Ailleurs, des amateurs de nature aspireront à séjourner dans des auberges qui offrent, à l'instar des établissements sélects du XIX siècle, à la fois le luxe de l'hébergement haut de gamme et le dépaysement d'un cadre rustique.

Quintessence de la sauvagerie, la forêt québécoise est par ailleurs mobilisée aux fins du développement du tourisme international, notamment de souche européenne. Bien que la superficie de la forêt se soit considérablement accrue dans presque tous les pays d'Europe depuis le début du XIXe siècle, et même si des expériences ont actuellement cours pour y réintroduire des animaux sauvages, notamment le loup et l'ours, cette forêt est généralement perçue comme le produit d'un jardinage plutôt que comme un phénomène naturel. Les débats que suscite la réinsertion d'espèces sauvages montrent incidemment que plusieurs s'accommodent de cette sylve domestiquée. La forêt québécoise, de prime abord peu fréquentable d'un point de vue européen, est pour cela même présentée comme le lieu d'un véritable retour à la nature... pourvu que les forestiers soient tenus à distance respectable.

Mais la fréquentation de la forêt ne se conjugue pas sur le seul mode des vacances ou du séjour prolongé. Depuis la fin des années 1960, le développement de la sensibilité environnementale a en effet engendré une demande croissante pour des milieux naturels accessibles en fonction d'une fréquentation quotidienne. Dans la région montréalaise, la création des parcs-nature de la défunte Communauté urbaine de Montréal, des parcs provinciaux d'Oka et du mont Saint-Bruno, de même que l'ouverture du centre de la nature du mont Saint-Hilaire, participent d'un mouvement auquel aucune agglomération urbaine d'Europe ou d'Amérique du Nord ne semble avoir échappé. Alors que la plupart des grands parcs de la seconde moitié du $X I X e$ siècle et du début du XXe ont été absorbés par l'urbanisation, la demande sociale s'est faite de plus en plus pressante pour que les bois des aires métropolitaines soient protégés et versés au domaine public. Un mouvement qu'avaient anticipé, en première moitié du XXe siècle, les concepteurs européens de ceintures vertes métropolitaines ou les créateurs du parc de la Gatineau, dans l'Outaouais québécois.

\section{L'arbre-équipement et la banalisation de la forêt}

Mais, dans ces aires d'influence métropolitaines, l'espace que la forêt-refuge entend soustraire à l'urbanisation ou soutirer à celui de la forêt-ressource, elle le dispute de plus en plus à de la forêt-ludique. On assiste en effet, depuis peu, à la multiplication des parcs de parcours déployés entre les cimes des arbres, de sites de "paintball sportif », de sentiers pour VTT ou bicyclette de montagne et d'autres installations similaires.

Si ces activités offertes en milieu forestier s'articulent parfois à la mémoire des lieux, par exemple en tirant parti des légendes qui y sont rattachées, comme à Sherwood, plus souvent qu'autrement, elles n'y cherchent qu'un environnement propice au déploiement d'installations récréatives, tantôt à caractère familial, tantôt de type sports extrêmes. Dans ces circonstances, un tel

aménagement récréatif [...] ne peut être que l'application standardisée de modèles techniques élaborés par les professionnels du tourisme. Le développement touristique de la forêt apparaît comme l'imposition d'équipements récréatifs dont I'utilisation répond à une logique instrumentale, mais ne repose sur aucune pratique de parcours, d'accumulation, de notations et de connaissances intimes de la forêt, fut-elle artistique (Kalaora, 1998 : 33).

La forêt n'y est pas valorisée pour ses caractères historiques, esthétiques ou écologiques intrinsèques, mais simplement comme support à des équipements qu'au demeurant, on pourrait installer ailleurs. En faisant de l'arbre un équipement de jeu ou un élément d'un dispositif favorisant le jeu, on banalise la forêt. Elle n'a conséquemment d'intérêt que si la rentabilité économique de l'activité reste supérieure à celle d'activités de substitution. 


\section{Encadré}

Nommer pour mieux domestiquer

La domestication de la forêt n'est pas qu'affaire d'aménagement; elle passe aussi par le langage. Chez les Celtes, le nemeton est la clairière sacrée ouverte dans les bois. Chez les Romains, familiers avec les champs (ager), ainsi que les vergers et les vignobles (hortus), le sultus identifie l'assemblage de landes et de bois (silvae). Au haut Moyen Âge, foresta (dont dérivent les appellations forêt, forest, forst) a d'abord une portée juridique ; il désigne une appropriation royale. Cette confiscation permet aux monarques de se réserver la silva regio (forêt profonde), dont une partie est dévolue au rituel de la chasse royale (silva forestis). Destinées à l'exploitation de la ressource, la silva concida fournit le bois de chauffage, tandis que la silva palaria fournit piquets, palissades ou manches d'outils. Ces forêts jardinées prendront le nom de taillis au Xle siècle. Âgées d'au moins 30 ans, les futaies sont pour leur part aménagées pour produire le bois d'œuvre. Cette réservation s'accompagne par ailleurs de la concession de droits d'usages de parties de la forêt aux manants et aux vilains ; on crée à cet effet la silva communis.

Au Québec, ces dénominations associées au statut juridique et à la vocation sont peu usitées. Si certains termes désignent un état particulier, par exemple brûlis (étendue de forêt ravagée par un incendie) ou chablis (étendue de forêt détruite par le vent, le verglas), les appellations tiennent davantage de la composition arborescente (érablière à caryer, pessière, etc.). Par ailleurs, la multiplication des termes liés à l'exploitation (coupe à blanc, de jardinage, de régénération, de succession, éclaircie, éclaircie commerciale, etc.) témoigne de la prépondérance du régime productiviste.

\section{Conclusion}

En Occident, l'invention de la forêt s'est déclinée sur plusieurs registres dont, à compter de la première moitié du XIXe siècle, ceux du loisir et du tourisme. II en a résulté une nouvelle catégorisation géographique qui a fait des massifs soumis à une double artialisation des territoires d'exception. Malgré tout, l'exploitation économique de la matière ligneuse reste globalement prépondérante, voire trop souvent exclusive. On ne s'étonnera donc pas que les attentes sociales ne soient que très partiellement satisfaites.

Mais les conflits d'usages ne sont pas attribuables à la seule subordination des usages sociaux aux usages économiques.
La mise en valeur de la forêt est aussi confrontée aux impacts d'une fréquentation qui s'apparente dorénavant, en certains lieux et en certaines circonstances, à une véritable invasion. Si, parce qu'elle « cristallise les fantasmes d'une nature sauvage idéalisée par une société majoritairement urbaine » (Boutefeu, 2005 : 11), la forêt est devenue une destination de plus en plus prisée, elle est par ailleurs, pour bon nombre d'écologistes, un archétype d'une nature qui doit être protégée de ce déferlement de visiteurs. Mais l'argumentaire écologique, prétexte à la fixation objective d'une capacité de charge, voile parfois, sous couvert d'écotourisme ou de tourisme durable, une motivation sociale, l'élite " environnementalement " sensible d'aujourd'hui prenant le relais de l'élite cultivée d'hier. Un fait que la surreprésentation des experts naturalistes dans les organisations responsables de la gestion des espaces protégés tend à occulter, par propos scientifiques interposés.

Ailleurs, les habitats pavillonnaires en forêt qui favorisent, tant par leurs cadres bâtis que paysagers, un rapport en apparence direct à une nature valorisée pour ses qualités intrinsèques, se sont multipliés ces dernières années. Ils réalisent en quelque sorte le rapatriement hic et nunc d'une jouissance édénique que garantiraient des restrictions d'occupation et de fréquentation qui verrouillent ces positions et sont présentées, par ceux qui en sont les uniques bénéficiaires, comme un moyen légitime d'en assurer la protection. Dans ce cas, les conflits d'usages ne sont pas seuls en cause. Malgré les apparences, ces paradis verts le sont souvent moins qu'on pourrait le croire, en particulier sur les littoraux forestiers - par exemple au Portugal ou sur la côte du Pacifique -, où des phénomènes comme les incendies prennent des allures de désastres environnementaux parce que ces forêts, transformées en lotissements résidentiels exclusifs, ont été rendues vulnérables pas le pompage excessif des nappes d'eau souterraines.

Bien que tentante, la réduction des conflits d'usage dans les forêts à une incompatibilité persistante entre ses usages sociaux, d'une part, et ses usages économiques, d'autre part, est donc difficilement conciliable avec ce qu'est devenue la forêt dans nos sociétés. Seul le dépassement de cette contradiction héritée des Lumières nous permettra de participer à la poursuite d'une invention fondatrice de l'Occident.
Gérard Beaudet est urbaniste, professeur titulaire et directeur de l'Institut d'urbanisme à l'Université de Montréal.

\section{Notes}

1 Les Athéniens se sont montrés préoccupés par la déforestation dès le VIe siècle av. J.C. Les correctifs envisagés ne furent toutefois pas suffisants pour remédier à la situation. Les enseignements qui auraient pu être retirés n'ont pas empêché les Romains d'exploiter parfois abusivement les territoires conquis, en particulier ceux du pourtour de la Méditerranée, qui ont durablement subi les conséquences des déboisements excessifs (Wright, 2006 : 127ss).

2 Cette forêt, la plus ancienne des enclaves créées par un souverain anglais, est aussi la plus connue et la plus belle de l'Angleterre. Sa notoriété lui a valu la publication, en 2000, d'un bestseller simplement intitulée The Forest (Rutherfurd, 2001).

3 En se portant acquéreur, en 1895, de ce joyau du golfe Saint-Laurent que constitue I'île d'Anticosti, l'industriel français Menier reprend en quelque sorte là où avaient laissé les souverains du Moyen Âge et leurs successeurs. L'asservissement d'un village dont la population est tout entière dévouée au maître du lieu, l'introduction d'espèces animales associées à la sauvagerie (ours noir, orignal, chevreuil, bison) et l'adoption de règlements extrêmement sévères destinés à contrôler toute forme d'exploitation des ressources de l'île constituent en effet une réédition d'une pratique qui avait significativement marqué la géographie forestière et la culture européennes. Le rêve fut toutefois de courte durée. Depuis 1974, l'île est une réserve faunique.

4 La pénurie est telle que la France se réserve certains arbres des forêts laurentiennes, dont les chênes. Cette mesure ne peut toutefois suffire à garantir les approvisionnements.

5 Lorsque les Français réalisent leurs premières incursions dans la vallée du Saint-Laurent, ils sont confrontés à un couvert forestier qu'avaient à peine entamé les quelques groupes amérindiens qui pratiquaient la culture sur brûlis. En conséquence, ils sont obligés de dégager des clairières partout où ils s'installent à demeure. II en est de même pour les colons de la Nouvelle-Angleterre. Malgré une colonisation somme toute relativement récente, de vastes superficies défrichées du Québec et de la Nouvelle-Angleterre sont reconquises par la forêt. Nés à la suite de déboisements déraisonnables de milieux impropres à la culture, plusieurs rangs des aires de colonisation tardive du Québec subissent incidemment un tel sort.

En revanche, plus de 1000 ans av. J.C., les Mayas et d'autres sociétés précolombiennes 
d'Amérique centrale avaient gagné sur la forêt d'immenses domaines voués à l'agriculture. Mais, à l'instar de la civilisation romaine, ces dernières n'ont pas su éviter le piège de la surexploitation du milieu. L'effondrement de cette civilisation fut suivi, bien avant l'arrivée des Espagnols, d'un reboisement inexorable qui envahit à nouveau les espaces qui avaient été soutirés à la forêt (Wright, 2006 : 135ss).

6 On peut lire le poème sur le site: [http://perso. orange.fr/alain. perron/Coureurdesbois.htm].

7 Adeptes du VTT (véhicule tout-terrain).

\section{Bibliographie}

Beaudet, Gérard (1999), "Paysages et investissement de valeur ", dans Philippe PoullaouecGonidec, Michel Gariépy et Bernard Lassus (dir.), Le paysage, territoire d'intentions, L'Harmattan, p. 35-54.

Beaudet, Gérard, et Serge Gagnon (1999) «Esquisse d'une géographie structurale du tourisme et de la villégiature: L'exemple du Québec ", dans Normand Cazelais, Roger Nadeau et Gérard Beaudet (dir.), L'espace touristique, Presses de I'Université du Québec, p. 133-195.
Boutefeu, Benoît (2005), «L'aménagement forestier en France: À la recherche d'une gestion durable à travers I'histoire ", Vertigo, vol. 6, n² 2 , [http://www.vertigo.uqam.ca/vol6no2/art21 vol6no2/vertigovol6no2_boutefeu.pdf].

Denecourt (1839), Guide du voyageur dans la forêt de Fontainebleau (cité dans [http://www.aaf.org/]).

Desmarais, Gaëtan (2006), «La structuration morphologique de la Rome antique, du centre organisateur à la configuration de seuil ", Espaces et sociétés, nº 122, p. 51.

Fenimore Cooper, James (1992), Le dernier des Mohicans, G-F Flammarion.

Foisy-Geoffroy, Dominique (2004), Esdras Minville: Nationalisme économique et catholicisme social au Québec durant l'entre-deux-guerres, Septentrion.

Germain, Georges-Hébert (2003), Les coureurs des bois, Libre Expression.

Harrison, Robert (1992), Forêts ; essai sur l'imaginaire occidental, Champs Flammarion.

Kalaora, Bernard (1998), Au-delà de la nature l'environnement, L'Harmattan.

Larrère, Raphaël et Olivier Nougarède (1993), Des hommes et des forêts, Découverte Gallimard.

Mariage, Thierry (2003), L'univers de Le Nostre, Pierre Mardaga.
Martin, Paul-Louis (1990), La chasse au Québec, Boréal.

Mercier, Guy (1998), "La quête urbaine de la nature: Matériau, gestion, mythe », dans Guy Mercier et Jacques Bethemont (dir.), La ville en quête de nature, Septentrion, p. 239-247.

Ritchot, Gilles (1998), « La ville en quête de valeur et le phantasme de la nature", dans Guy Mercier et Jacques Bethemont (dir.), La ville en quête de nature, Septentrion, p. 65-73.

Roger, Alain (1997), Court traité du paysage, Gallimard.

Rutherford, Edward (2001), The Forest, Ballantine Books.

Schama, Simon (1999), Le paysage et la mémoire, Seuil.

Solnit, Rebecca (2002), L'art de marcher, Actes Sud.

Thoreau, Henry David (2003) De la marche, Mille et une nuit.

Thoreau, Henry David (1982) Walden ou la vie dans les bois, Aubier.

Viard, Jean (1990), Le tiers espace; essai sur la nature, Méridien Klincksieck.

Wright, Ronald (2006), Brève histoire du progrès, $\mathrm{HMH}$, coll. "Constantes ».

www - uqam ·ca

\section{Programmes de deuxième cycle en Gestion et planification du tourisme}

- Diplôme d'études supérieures spécialisées (D.E.S.S.) offert à temps partiel, 2ans

- Maîtrise (M.Sc.) offerte à temps plein ou partiel, 2 ou 4 ans

Conditions générales d'admission :

Détenir un diplôme de premier cycle et deux ans d'expérience pertinente

Informations: Denyse Ste-Marie (514) 987-3000 poste 4750

http://www.programmes.uqam.ca/3741 http://www.programmes.uqam.ca/3041

Gestion du tourisme international et des services d'accueil

- MBA pour cadres offert à temps partiel uniquement, 2 ans

Conditions générales d'admission :

Détenir un diplôme de premier cycle et occuper un poste cadre en gestion depuis au moins quatre ans

Informations: Marie-Hélène Vaillancourt (514) 987-3000 poste 2538

http://www.esg.uqam.ca/mbatourisme/

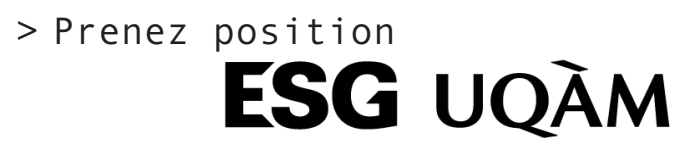

